\title{
Téoros
}

Revue de recherche en tourisme

\section{Gais et lesbiennes en voyage}

Une rencontre avec les droits humains dans le monde

\section{Roberto Jovel}

Volume 19, numéro 2, été 2000

Le tourisme des gais et des lesbiennes

URI : https://id.erudit.org/iderudit/1071957ar

DOI : https://doi.org/10.7202/1071957ar

Aller au sommaire du numéro

\section{Éditeur(s)}

Université du Québec à Montréal

\section{ISSN}

0712-8657 (imprimé)

1923-2705 (numérique)

Découvrir la revue

\section{Citer cet article}

Jovel, R. (2000). Gais et lesbiennes en voyage : une rencontre avec les droits humains dans le monde. Téoros, 19(2), 6-10. https://doi.org/10.7202/1071957ar d'utilisation que vous pouvez consulter en ligne.

https://apropos.erudit.org/fr/usagers/politique-dutilisation/ 


\section{Gais et lesbiennes en voyage}

\section{UNE RENCONTRE AVEC LES DROITS HUMAINS DANS LE MONDE}

\section{Roberto Jovel}

Imaginez-vous, voyageur gai québécois, voyageuse lesbienne québécoise en situation d'immersion en milieu gai et lesbien lors d'un séjour à l'étranger. Au Pérou, par exemple, «Malgré l'adversité à laquelle font face les minorités sexuelles, il y existe une sorte de camaraderie et de protection mutuelle très fortes entre les personnes qui fréquentent un établissement quelconque, même si elles ne se connaissent pas. Ce, bien entendu, 'en respectant' l'appartenance de chacun à une strate déterminée ainsi que les valeurs générales de la société ", raconte Jorge Flores, membre du Réseau de soutien des droits des gais et des lesbiennes d'Amnistie Internationale, section canadienne francophone.

Des paradoxes comme celui-là étonneront le voyageur ${ }^{1}$ : cette solidarité spontanée jaillit dans les lieux de rencontre $\operatorname{lgbt}^{2}$ en suivant les contraintes normatives de « l'extrême stratification socio-économique unie au racisme, qui est basé sur les différentes nuances de peau brune $»$. Mais l'adversité soulevée par ce témoignage, de quoi a-t-elle l'air ? « La furieuse homophobie ambiante et la 'protection de la vie privée' de la clientèle, à 99,9\% encore dans la garde-robe, font en sorte que les établissements gais et lesbiens demeurent dans la clandestinité ». C'est qu'une telle adversité se manifeste par les biais les plus insidieux : « le machisme et l'homophobie se retrouvent au sein même du milieu gai, ce qui s'exprime par le rejet envers les personnes qui s'écartent des normes de genre établies et par le rejet et la méfiance envers celles qui affirment publiquement leur orientation sexuelle ». Ces témoignages font état d'une situation sociale, culturelle et politique écrasante : un long chemin reste à faire avant de s'affranchir de la domination qu'exercent l'hétérosexisme et l'homophobie au Pérou. Ceci sans évoquer la discrimination quotidienne, la persécution et la répression qui persistent, en dépit de la non-existence de lois criminalisant l'homosexualité. Le touriste a-t-il à craindre cette situation ? A-t-il à en apprendre quelque chose ? Peutêtre. Sûrement, la combinaison d'une bonne dose de prudence et d' autant de solidarité pourrait se révéler l'attitude idéale quand on se rend ailleurs en visite ou en vacances. lecte de renseignements habituels, le voyageur s'arrêtera aux passages des guides touristiques lgbt qui portent sur les aspects légaux et culturels qui encadrent l'homosexualité dans le pays où il est sur le point de se rendre : il trouvera toujours un paragraphe très général qui met le touriste en garde et l'oriente quant à l'attitude qu'il doit adopter sur place, question d'éviter des ennuis, des surprises désagréables, des problèmes majeurs. On conseille aussi la consultation des sources d'information spécialisées en matière de droits humains des minorités sexuelles. Il existe de la documentation sous diverses formes, depuis les recensions des situations légales dans chaque pays jusqu'aux études les plus pointues concernant des as-
Après le choix de la destination et la col- pects socioculturels des lgbt sous diverses latitudes. Une visite aux sites Internet de l'AILGBT, de l'ILGA et de l'IGLHRC ${ }^{3}$ vous permettra de vous sensibiliser à l'avance au sort des lgbt, à leur vie quotidienne. Si, en effet, l'on peut décider de boycotter une destination où le système politique est particulièrement hostile aux lgbt, on peut également décider d'y aller, de rencontrer des gens et d'apprendre quelque chose sur ce que c'est qu'être lgbt ailleurs dans le monde.

\section{PRÈS D'ICI, LES “DESTINATIONS SOLEIL "}

Récemment, un journaliste demandait à Amnistie des renseignements concernant la situation des droits humains des minorités sexuelles à Cuba. Bien qu'on puisse $\mathrm{y}$ constater des avancements très positifs, des problèmes importants restent à résoudre. L'époque est révolue où le régime de ce pays caraïbéen considérait les lgbt comme étant des ennemis de la Révolution et où on les confinait dans des « camps de rééducation » où ils se voyaient soumis à une combinaison de travaux forcés et d'endoctrinement censés les ramener sur le bon chemin. Puis, il y a eu cette ouverture, très publicisée par le régime et certes significative, à l'époque de Fraise et chocolat, film qui faisait effectivement la critique de l'ancienne attitude des communistes cubains à l'endroit des lgbt. Enfin, on a également assisté à la fin de cette affreuse politique qui consistait à renfermer les personnes séropositives ou vivant avec le sida en résidences spéciales, en retrait de la ville. Cela dit, il y a deux traits de la politique cubaine envers les minorités sexuelles qui préoccupent toujours le 
mouvement international pour les droits humains : d'abord, l'article 303 du Code pénal, qui prévoit une peine allant de trois mois à un an de prison pour des personnes qui manifestent publiquement leur homosexualité ; c'est la vieille notion de « scandale public » (Amnistie Internationale, 1998 : 78). Cet article est très explicite quant au groupe social visé, mais il reste peu clair quant aux conditions de son application, de telle sorte que la police fait à sa guise, l'arbitraire primant : il y a des descentes dans des lieux de rencontres lgbt, des arrestations, des amendes... Dans pareilles circonstances, le deuxième problème se révèle le plus grave : la liberté d'association et d'expression est inexistante, le système interdit toute forme d'organisation et de publication indépendantes. Ainsi, si 1994 voyait naître l'Association cubaine des gais et lesbiennes, en 1997 survenaient la répression et la fermeture de cette organisation non gouvernementale $^{4}$. La réponse des lgbt cubains en est une de survie : ils ont tissé un véritable réseau souterrain de contacts et d'information qui se montre en effet très solide et solidaire. Reste que, dans les faits, aucune possibilité n'existe pour les minorités sexuelles cubaines de prendre place et parole ouvertement dans l'espace public pour revendiquer, exiger, s'affirmer...

Mais la répression systémique basée sur des critères constitutionnels n'est pas le trait caractéristique des seuls régimes communistes. Au Panama, par exemple, en octobre 1999, le ministre de l'Intérieur et de la Justice, M. Winston Spadafora, refusait à l'Asociación Hombres y Mujeres Nuevos de Panamá (AHMNP, groupe communautaire lgbt du pays) l'octroi d'un statut juridique officiel. Les raisons? L'article 39 de la Constitution, les articles 64 et 69 du Code civil et d'autres articles de lois établissent clairement qu'il est interdit d'accorder un statut officiel à des associations dont les activités violeraient certains principes de « la morale » et des «bonnes mœurs $»^{5}$. Il en va de la distinction, chez les acteurs sociaux et politiques, entre l'affirmation des propres convictions et le principe démocratique du pluralisme des convictions : même les politiciens n'ont pas toujours appris le sens de cette distinction, ils restent en deçà d'une véritable culture démocratique basée sur les droits humains. Pis encore, il y a les pays où les lobbies d'extrême droite, la réaction ecclésiale et les partis politiques conservateurs fusionnent dans un seul pôle politique et social, très puissant, aux visées autoritaires en matière d'éthique sexuelle. À leurs yeux, leurs propres convictions devraient s'imposer à l'ensemble de la population. Par exemple, rappelons le Parti Action Nationale (PAN) au Mexique, organisation de droite aux origines très catholiques qui, se retrouvant à la mairie de Guadalajara il y a quelques années, a donné le coup d'envoi à des persécutions policières des gais, question d'assurer un « retour à la moralité », un « nettoyage moral » de la société. Des campagnes semblables, prenant appui sur les mœurs et les croyances ancestrales de la population, ont également été entamées par le PAN au Yucatán.

Au Mexique, des groupes communautaires lgbt existent depuis la fin des années 1970 et leurs prises de position sont souvent relatées dans les journaux. Au Salvador, par contre, où les médias sont monopolisés par une oligarchie extrêmement conservatrice sur le plan moral, être gai ou lesbienne reste - dans le cadre du discours médiatisé - une question de «perversion», de «péché », de «dysfonctionnement sexuel », de « maladie»... Mais au Salvador, comme un peu partout en Amérique centrale, les groupes communautaires lgbt se montrent de plus en plus en mesure d'agir, de se doter d'outils de développement social, d'interpeller les autorités tant locales que nationales et de contester le discours et les pratiques homophobes hégémoniques, de source le plus souvent religieuse. Prenons l'exemple du Costa Rica où les organisations non gouvernementales lgbt ont déjà parcouru un bout de chemin significatif en termes de lutte pour leurs droits. Il n'y a pas longtemps, un festival touristique lgbt était annulé par les autorités costaricaines après qu'un prêtre ayant tribune à la télévision ait dénoncé dans le cadre de son émission la prochaine tenue de l'événement gai. Aux propos « anti-lgbt » de ce prêtre s'étaient aussitôt ajoutées des déclarations publiques dans le même sens par l'Archevêque de San José et, comble de la situation, le président costaricain lui-même justifiait le geste discriminatoire et démantelait le festival touristique lgbt. L'association Triángulo Rosa a alors entamé une poursuite judiciaire contre le prêtre téléanimateur, contre l'archevêque et contre le président du pays, un dossier bien étayé à l'appui pour faire valoir chaque article et chaque paragraphe de la Déclaration universelle des droits de l'homme, des pactes, des conventions et des accords internationaux et des lois internes au Costa Rica qui étaient violés par 1'ordre officiel d'annulation du festival. C'est là ce qu'on appelle « connaître ses droits et s'en prévaloir politiquement ».

\section{LES DIFFÉRENCES RÉGIONALES, LES RAPPROCHEMENTS INTERNATIONAUX}

Si les groupes communautaires de l'Amérique latine font preuve, en général, d'un certain rapprochement par rapport à la politique et aux idées lgbt de l'Amérique du Nord et de l'Europe, cela n'est pas nécessairement le cas dans d'autres régions du monde ${ }^{6}$. Le défi qui se pose au voyageur, c'est de se reconnaître porteur en quelque sorte de «lunettes culturelles » et il lui faut avant tout écouter plutôt que juger, s'il veut mieux comprendre les personnes qu'il rencontre à l'étranger. Amnistie Internationale est consciente du risque impliqué à adopter une attitude « assimilationniste », pour ainsi dire, qui verrait des « gais » et des « lesbiennes » où il n'y en a pas. La diversité transculturelle - tant comportementale que symbolique - des rapports sexuels entre des personnes du même sexe biologique ne se laisse pas réduire à l'identification intime assortie de la présentation sociale de soi par l'entremise de signes très précis — en l'occurrence, « gai », « lesbienne », etc. - chargés de nombreuses connotations et, somme toute, issus d'une région culturelle particulière de la planète. Le document de campagne et de sensibilisation de l'AILGBT, intitulé Plus fort nous chanterons (1999), fait preuve d'un tel discernement, en prenant soin de ne pas coller aveuglement des étiquettes sur des réalités radicalement autres par rapport à ce que l'on connaît en Occident. Or, ledit document recueille en même temps les propos d'Ashok Row Kavi, militant indien qui, pour sa part, n'hésite point à affirmer : « Nous sommes une minorité [...] véritablement internationale. Dans de vastes régions de l'Afrique et de l'Asie, nous assistons à l'émergence de l'identité gaie » (Amnistie Internationale, 1999 : 15). C'est dire que, même dans des sociétés autres, la tendance se dessine avec de plus en plus de force, chez des gens dont la sexualité s'écarte des normes prescrites, à s'affirmer publiquement en clé identitaire : on 
en vient à la constitution d'agents politiques se nommant « lgbt» et exigeant d'être reconnus comme tels. Ces mouvements nouveau-nés, bien entendu, portent en leur sein toutes les variantes locales de la diversité sexuelle, mais ils établissent des liens de solidarité avec leurs contreparties occidentales, fusionnant dans un seul mouvement mondial. C'est dans l'équilibre entre les irréductibles particularités régionales et l'inévitable convergence planétaire que tout se joue.

Prenons alors le monde islamique. Un élémentaire souci de justice nous mène à mettre à découvert la tendance, trop répandue hélas, à croire que ce soit partout pareil dans cette aire géographique et culturelle et que la réalité locale corresponde à l'image caricaturale engendrée par le manque d'information rigoureuse, carence qui constitue un des grands problèmes actuels en matière de droits humains et de diversité sexuelle. Quelques distinctions s'imposent, d'abord sur le plan strictement juridique. C'est seulement dans certains pays, dont l'Afghanistan, l'Arabie Saoudite et l'Iran, que la Charia (loi islamique) s'applique, réservant aux homosexuels des châtiments allant des flagellations jusqu'à la peine de mort, sous diverses modalités : par exemple, en laissant tomber un mur de pierres sur l'accusé. Ou, encore, d'après 1'Ayatollah Musava Ardelsili, de 1'Université de Téhéran :

[...] il faudra se saisir de la personne, la maintenir debout, la partager en deux avec une épée et soit lui trancher la tête, soit la fendre en deux toute entière. Il (ou elle) tombera... Après sa mort, il faudra dresser un bûcher, placer le cadavre dessus, y mettre le feu et le brûler. Ou bien, l'emporter sur une falaise et l'y précipiter, puis les morceaux du cadavre devront être rassemblés et brûlés. Ou alors il faudra creuser un trou, y faire un feu et l'y jeter vivant(e). Nous n'avons pas de telles punitions pour d'autres crimes (Amnistie Internationale, 1998 : 32-33).

Dans d'autres pays, par contre, les peines varient : en Algérie, trois ans de prison (art. 338) ; en Libye, cinq ans (art. 407); au Maroc, trois ans (art. 489). En Égypte et en Irak, il n'y a pas de référence explicite à l'homosexualité dans les lois. Toutefois, même dans ces pays où la Charia n'a pas cours, l'éventualité de l'application

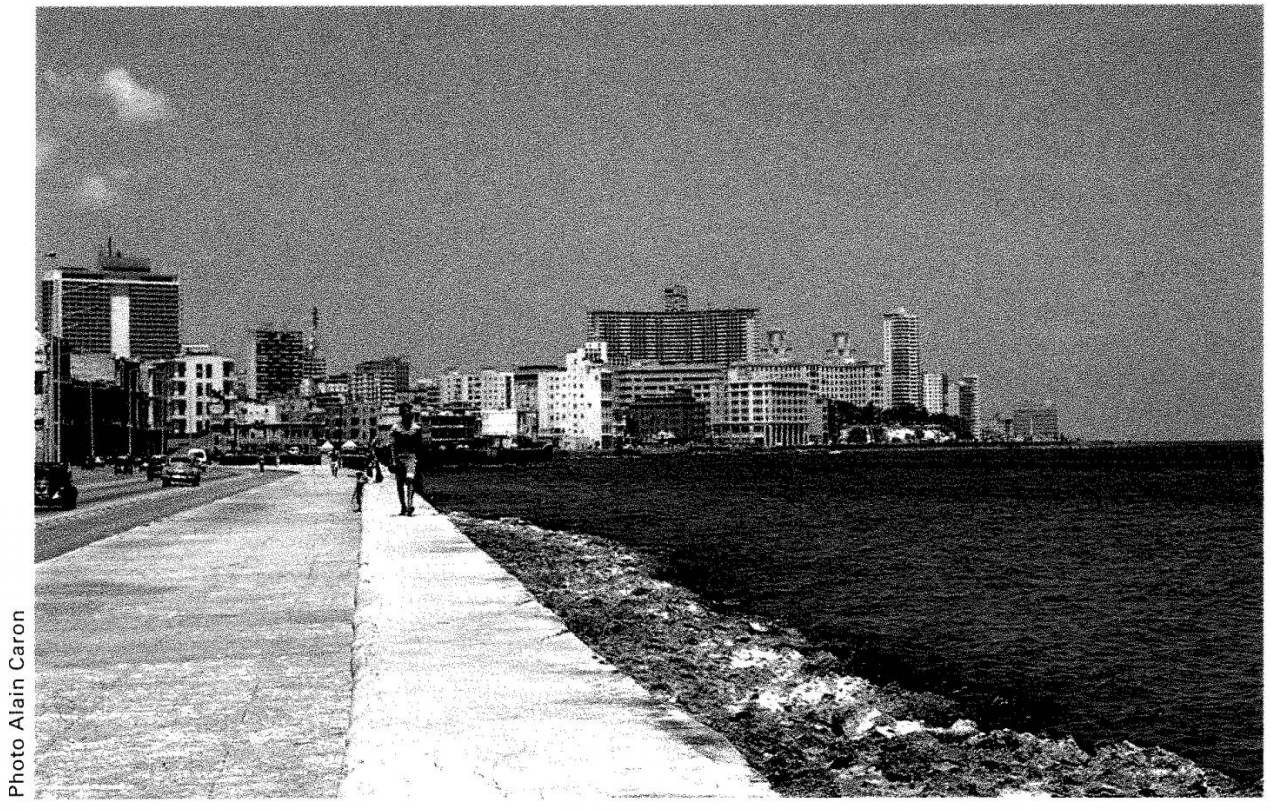

Le Malecón (bord de mer) havanais, comme celui des autres villes cubaines, est le lieu de rassemblement privilégié des gais, principalement le soir avant et après les fiestas « privées » organisées en banlieue.

d'autres peines et la crainte de l'éviction sociale maintiennent dans la clandestinité toute forme de vie impliquant une sociosexualité alternative ou de simples pratiques sexuelles différentes de la norme. La répression y est le plus souvent impitoyable, quoique, il est connu, dans certaines villes - les plus grandes et les plus touristiques, comme Casablanca - des niches de tolérance semblent prévaloir, avec des hauts puis des bas, pendant certaines périodes. Mais l'émergence d'une organisation lgbt telle que nous la connaissons ici est pour l'instant impensable dans le monde islamique. Peut-être l'espoir estil à retrouver chez les lgbt musulmans qui résident un peu partout à l'extérieur de leur région d'origine : nos meilleurs vœux vont ici à la Fondation A1-Fatiha, récemment créée aux États-Unis, et dont le travail se veut transformateur au sein même de la culture et de la spiritualité musulmanes (Alam, 2000). Entre-temps, il ne faut pas négliger le facteur « résistance culturelle » par rapport à l'uniformisation en provenance de l'Occident, le mouvement « postcolonialiste » aussi, très forts dans les pays islamiques. Il en va d'une volonté ferme de secouer le cadre culturel imposé par les puissances coloniales, en faveur d'un retour aux racines authentiques des nations en question. Programme légitime, sans doute, sur un certain nombre de plans, mais qui tombe dans un déni flagrant quant aux phénomènes homoérotiques s'étant depuis toujours manifestés dans l'histoire de ces peuples, même à l'époque où les musulmans se sont à leur tour trouvés dans la position des dominants par rapport à des sociétés européennes (Murray et Roscoe, 1997 : 142-157).

\section{UNE “ AUTHENTICITÉ » ADULTÉRÉE, QUI RÉPRIME SANS TRÊVE}

Ce retournement douteux des intentions décolonisatrices - autrement tout à fait légitimes - s'est également manifesté dans les événements récemment survenus en Afrique subsaharienne. Le conflit entre le président du Zimbabwe, le célèbre homophobe Robert Mugabe, et le gouvernement du Royaume-Uni en est 1'exemple le plus saillant, mais certainement pas le seul. Tout comme les présidents Moï du Kenya et Museveni de l'Ouganda, Mugabe voudrait faire croire au monde que la présence - très forte et aguerrie dans son pays - de personnes et de collectifs s'affirmant en tant que sujets porteurs d'une sexualité différente correspondrait à des réalités foncièrement étrangères à la culture africaine. Or, en même temps, Mugabe cite la Bible afin de rallier son peuple autour de sa politique discriminatoire et répressive face aux lgbt. Il reprend ainsi un discours de surface pour la décolonisation vis-à-vis des Britanniques, alors qu'il légitime et revendique cette autre colonisation toujours à l'œuvre au niveau religieux. Mais, 
tel que des militants et des professeurs africains n'ont pas tardé à le démontrer, il y a chez ces leaders politiques une confusion et une ignorance accablantes par rapport à l'histoire des sexualités en Afrique : l'existence de variations significatives de la sexualité au sein des cultures africaines précoloniales a été documentée et finement décrite (Murray et Roscoe, 1997). L'Afrique du Sud est l'exception qui mérite d'être saluée. Sa nouvelle constitution politique est exemplaire à bien des égards, mais aussi en ce qu'elle interdit explicitement toute discrimination basée sur l'orientation sexuelle. Bien entendu, la seule écriture de la loi ne change pas les vieilles attitudes du jour au lendemain : à preuve, le récent attentat à la bombe dans un bar gai de Cape Town.

Prenons maintenant l'Inde, cette culture de l'Asie du Sud où règne également le discours truqué de l'« authenticité ». Sous le critère des « actes contre l'ordre de la nature », l'article 377 condamne l'homosexualité (chez les hommes) à des peines qui incluent la flagellation et l'emprisonnement à perpétuité. Quant aux femmes, voici un témoignage éloquent :

Chez les lesbiennes, l'isolement et l'invisibilité ont entraîné l'absence d'une identité collective. Dans le domaine public, il n'y a pas de place pour la discussion sur la sexualité, encore moins sur la sexualité des femmes. La culture, la tradition, les valeurs et la morale ont été systématiquement utilisées comme dispositifs d'oppression et de discrimination. Dans ce cadre, nous sommes marginalisées en tant que femmes et, doublement, en tant que lesbiennes... Cela doit-il surprendre, alors, qu'il n'y a que très peu de lesbiennes qui $s$ 'affirment publiquement en Inde $e^{7}$ ?

Mais le déni contemporain du lesbianisme en ce pays, faisant valoir une supposée pureté culturelle, a été efficacement remis en question et critiqué par Giti Thadani, jeune Indienne qui s'est consacrée à de longues années de recherches, notamment sur des sites archéologiques de son pays ${ }^{8}$. Elle y a repéré les statues, les bas-reliefs en pierre qui représentent des scènes d'intimité corporelle entre femmes. Elle a écrit un livre sur le sujet qui n'a pu être édité qu'à l'extérieur de son pays. Or, comme nous l'avons soulevé précédemment, en Inde puis au sud de l'Asie en général, la diversité sexuelle se manifeste dans la population sous des formes très variées qui dépassent le cadre des termes « gai » et «lesbienne » et qui s'en accommoderaient difficilement ${ }^{9}$. Au Pakistan, par exemple, des pratiques sexuelles sont souvent entretenues entre des hommes mariés et des jeunes garçons, suivant un certain nombre de contraintes et de modèles précis, de sorte que, si de telles pratiques sont gardées sous silence, toute la structure sociale de la famille se maintient intacte. Et on ne remet pas nécessairement en question l'attirance que de tels hommes mariés ressentent pour les femmes, encore moins leur propre identification comme mâles hétérosexuels... Bien que les lois pakistanaises prévoient des peines semblables à celles en cours en Inde, elles sont rarement appliquées, les procédures nécessaires à leur mise en œuvre étant fort complexes et compromettantes pour les éventuels témoins de ces «crimes » (Murray et Khan, 1997).

En Extrême-Orient, la situation varie énormément aussi, selon qu'on se déplace des Philippines au Japon, en Corée ou en Thaïlande... En Chine, par exemple, 1'homosexualité est légale, mais elle est parfois poursuivie en vertu de l'article 106 qui prévoit une peine de cinq ans de prison pour «trouble de l'ordre social ». La Mongolie voyait récemment une première organisation lgbt être mise sur pied au centre ville d'Ulan Bator, sous le nom de «Tavilan» (Destinée), dans le but de contrer le harcèlement policier et l'imposition de sentences qui violent les droits civils. Disons ici pour l'instant que, dans les pays où les actes homosexuels sont criminalisés, l'accusation d'homosexualité - qu'elle soit fondée ou non - peut être utilisée à des fins oppressives qui violent les droits humains. Prenons le cas flagrant de la Malaisie, où ce genre de démarche a récemment été entrepris par le premier ministre Mahathir dans le but d'annihiler politiquement son vice-premier ministre. Anwar Ibrahim s'est fait arrêter par la police, a été roué de coups et soumis à un procès inéquitable, à l'encontre des standards internationaux. Détenu depuis septembre 1998, il risque une peine d'emprisonnement de vingt ans et le fouet. ${ }^{10}$

Et le «Premier Monde», comment se porte-t-il ? En voici quelques exemples. $\mathrm{Si}$ vous pratiquez la «sodomie» entre hommes en Arkansas, au Kansas, en Oklahoma, au Texas ou au Missouri, vous êtes considéré par les autorités comme un criminel. Si vous aviez été en vacances à Londres le 30 avril 1999, en train de prendre un verre au Admiral Duncan Pub, vous auriez été victime d'un attentat à la bombe revendiqué par des fanatiques d'extrême droite. Si vous êtes un jeune gai de moins de dix-huit ans en Autriche et que vous faites l'amour avec un autre jeune homme, vous risquez de vous retrouver en institution pénitentiaire, alors que le reste de la population peut vivre sa sexualité librement à partir de l'âge de quatorze ans. Les lgbt de l'Écosse se font comparer aux nazis par le cardinal d'Édimbourg qui, par ailleurs, met tout le poids de son influence à contribution pour maintenir en vigueur le paragraphe 28 de la Loi écossaise qui interdit aux écoles publiques de traiter l'homosexualité comme quelque chose de légitime...

\section{PRENDRE LE POULS LORSQU'ON EST EN VOYAGE : LES PAYS-BAS ET L'ESPAGNE}

En vacances récemment aux Pays-Bas, j’ai pu prendre le pouls de la situation des lgbt dans ce pays qui se présente souvent, et avec raison, comme à l'avant-garde en matière de tolérance, de respect, d'ouverture à la diversité, qu'elle soit religieuse, idéologique ou sexuelle. D'après les témoignages des militants, certains effets de l'immigration comporteraient un véritable recul, particulièrement en ce qui concerne l'éducation des adolescents où les Néerlandais avaient pourtant réussi à instaurer une atmosphère ouverte et une approche sensible aux différences sexuelles. De jeunes nouveaux arrivants boycottent carrément les démarches pédagogiques en matière d'orientation sexuelle : ils interrompent sans cesse, ils sèment le désordre et la révolte, ils insultent... D'aucuns en sont venus même à bousculer des professeurs, les salles de classe devenant ainsi de véritables otages de cette homophobie insoumise qui ne s'adapte guère aux politiques locales. Cela ne peut que rappeler des situations qui se produisent toujours, au Québec, dans le cadre des Centres d'orientation et de francisation pour immigrants (COFI) : même si les politiques officielles dans le milieu se sont beaucoup améliorées au cours des derniers cinq ans, des dynamiques homophobes continuent de se déchaîner parmi les étudiants, allant de l'insulte et du lynchage 
moral jusqu'à l'ostracisme total, ce qui mène des nouveaux arrivants gais et lesbiens parfois même à la dépression.

Le thème de l'éducation des jeunes aux réalités lgbt était aussi à l'ordre du jour lors de mon passage en Espagne. Le Colectivo de Lesbianas y Gays de Madrid (COGAM) venait, en janvier 2000 , de publier un manuel didactique intitulé 25 questions sur l'orientation sexuelle, instrument de formation pour enseignants et intervenants au niveau des écoles secondaires. Édité en partenariat par le COGAM, l'Assemblée de Madrid et le Protecteur du mineur luimême, le manuel a été distribué dans les écoles madrilènes en vue de consolider les principes de non-discrimination et de valorisation de la diversité - y compris sexuelle - prévus par la Loi générale de l'éducation de l'État espagnol. Or, des réactions enragées se sont vite fait entendre de la part de certains groupes de pression religieux à la suite de la mise en circulation de ce matériel pédagogique : celuici ferait la promotion de l'homosexualité et inciterait les jeunes à la promiscuité... La communauté lgbt québécoise aura intérêt à suivre ce dossier, la question de l'éducation en matière de diversité sexuelle restant toujours à aborder et à résoudre au Québec ${ }^{11}$, compte tenu surtout des barrières que de puissants secteurs religieux conservateurs continuent de dresser avec succès à cet égard, notamment à la Commission scolaire de Montréal ${ }^{12}$.

\section{CONCLUSION}

Les lesbiennes, les gais, les bisexuel(le)s, les travesti(e)s et les transsexuel(le)s... toutes les personnes dont la sexualité ne s'en tient pas aux strictes prescriptions des systèmes hétérosexistes, vivent sous des régimes éthico-politiques assez variés dans diverses régions du globe. Souvent l'épanouissement - personnel, social - leur est refusé avec profusion de violence, par les moyens les plus inhumains. Mais les membres de ces collectivités minoritaires résistent quotidiennement, gagnent des forces, se font une vie à contre-courant. Se rendre sur place - chez eux, chez elles-en séjour de vacances représente pour le voyageur québécois à chaque fois une occasion unique pouvant lui permettre d'accéder à un univers culturel autre que le sien propre. Une attitude sensible dépassant les bornes de notre propre manière de vivre et de comprendre le monde est- elle envisageable, une telle ouverture vers l'autre, qui nous enrichisse par les apprentissages qu'elle entraîne, susceptibles de nous transformer, est-elle possible ? Et le témoignage - hors de tout paternalisme facile et insultant - de notre propre démarche historique en tant que collectivité lgbt québécoise, peut-il à son tour se révéler utile et pertinent pour les gens d'ailleurs? Ce dialogue interculturel contribue à tisser des liens qui brisent l'isolement : plus nous nous tiendrons en contact étroit, plus nous réussirons à nous affranchir de l'oppression en tant que communauté planétaire. Ces lesbiennes et ces gais foncièrement autres sont tout de même et sans conteste nos semblables : des consœurs et des confrères qui, lorsque nous les visitons, nous rappellent que notre propre situation minoritaire prévaut partout. Ils nous rappellent le visage terrible de cette discrimination et de cette répression que nous avions probablement déjà - trop tôt, trop rapidement — oubliées, estimant qu'elles étaient chose du passé.

Roberto Jovel, détenteur d'un baccalauréat en sociologie de l'Université ARCIS (Santiago de Chili) et en philosophie de l'Université du Québec à Montréal, poursuit actuellement un doctorat en sémiologie au sein de cette même université. Il est coresponsable du Réseau de soutien des droits des lesbiennes, gais, bisexuel(le)s et transsexuel(le)s d'Amnistie Internationale, section canadienne francophone.

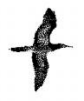

\section{NOTES}

1 Dans le seul but d'alléger le texte, nous nous servirons en général du masculin dans son sens neutre. De même, lorsque nous parlons de « gais et lesbiennes », nous entendons faire référence à toutes les personnes dont la sexualité ne se conforme pas à la norme hétérosexiste.

2 Le sigle «lgbt» signifie : lesbiennes, gais, bisexuel(le)s, travesti(e)s et transsexuel(le)s.

3 Le Réseau AILGBT canadien francophone : www.algi.qc.ca/asso/amnistie.html ; International Lesbian and Gay Association (ILGA) : www.ilga.org ; International Gay and Lesbian Human Rights Commission (IGLHRC) : www.iglhrc.org

4 Référer à l'enquête mondiale, réalisée par l'ILGA, sur la situation légale et politique des lgbt (www.ilga.org)

5 Décision \# 448-R-118, Ministère de l'Intérieur et de la Justice, Panama, 5 octobre 1999.

6 Le constat de ce rapprochement au niveau du politique ne se veut point un nivellement des différences culturelles : rappelons ici le cas des muxes, ces individus de l'isthme de Tehuantepec, au Mexique, porteurs d'une identité sexuelle (y compris un rôle de genre et des pratiques sexuelles) toute particulière et irréductible. Ce n'est là qu'un cas frappant parmi beaucoup d'autres nuances repérables dans la région.

7 Propos d'une membre du groupe communautaire indien « Campaign for Lesbian Rights », cités dans Amnistie Internationale, 1999 : 6.

8 AILGBT Pays-Bas (et coll.), Hors la loi, production audiovisuelle concernant les droits humains des lgbt dans cinq pays du monde.

9 Pour un bel exemple de cette diversité irréductible dans le contexte de l'Asie du Sud, référer à l'article de Kumar, Arvind, « Hijras: Challenging Gender Dichotomies », dans Ratti, 1993 : 85-91

10 Le Dire!, janvier-mars 2000.

11 Un texte qui traite en profondeur de la situation de cette démarche en Espagne se trouve dans Le Dire !, juillet-septembre 2000.

12 Le Dire!, avril-juin 2000

\section{BIBLIOGRAPHIE}

Amnistie Internationale (1998), Briser le silence, Paris.

Amnistie Internationale (1999), Plus fort nous chanterons, Paris.

Alam, Faisal (2000), « La situation actuelle de la communauté musulmane gaie et lesbienne et les perspectives pour l'avenir », dans Le Dire!, dienne francophone, juillet-septembre.

Murray, Stephen O., et Badruddin Khan (1997), "Pakistan ", sous la direction de Donald J. West et Richard Green, Sociolegal Control of Homosexuality, Plenum Press, New York, 1997.

Murray, Stephen O., et Will Roscoe (sous la direction de) (1997), Boy-Wives and Female Husbands. Studies in African Homosexualities, St. Martin's Press, New York.

Murray, Stephen O., et Will Roscoe (sous la direction de) (1997), Islamic Homosexualities, New York University Press. En particulier, l'étude de Louis Crompton, « Male Love and Islamic Law in Arab Spain », p. 142-157.

Ratti, Rakesh (sous la direction de) (1993), A Lotus of Another Color, Alyson Publications Inc., Boston. bulletin du Réseau AILGBT de la section cana- 